\title{
Tratamento Anti-Hipertensivo. Prescrição e Custo de Medicamentos. Pesquisa em Hospital Terciário
}

\author{
Daniela Akashi, Flávia K. Issa, Alexandre C. Pereira, Anna C. Tannuri, Daniele Q. Fucciolo, \\ Maurício L. Lobato, Tatiana G. Galvão, Isabela M. Benseñor, Paulo A. Lotufo
}

São Paulo, SP

\begin{abstract}
Objetivo - Verificar os medicamentos anti-hipertensivos mais utilizados por pacientes que procuram atendimento em hospital público terciário, avaliando o impacto das diretrizes de atendimento (consensos) e custo de aquisição.

Métodos - Foram selecionados 141 pacientes (101 do sexo feminino) de 40 a 72 (média 53,3) anos, que procuraram de forma espontânea, atendimento em hospital terciário, com diagnóstico prévio de hipertensão arterial feito por médico e ausência de queixas relacionadas ao aparelho cardiovascular.

Resultados - Verificou-se que 75,9\% $(n=107)$ estavam em uso diário de anti-hipertensivos, sendo 60,7\% $(n=86)$ em monoterapia e os demais em terapia mista. Os medicamentos mais empregados em monoterapia eram: tiazídicos, metildopa, inibidores da ECA, bloqueadores de canal de cálcio e betabloqueadores. A combinação com tiazídicos (26,3\% do total) seguiu a mesma preferência. O segundo medicamento mais prescrito, metildopa, era o de maior custo. Metade dos pacientes adquiriu os medicamentos por compra direta.

Conclusão - Observou-se maior utilização de antihipertensivos de alto custo, conduta discordante das principais diretrizes das sociedades médicas, sobretudo do $V$ $J N C$, que preconizou tiazídicos e betabloqueadores, como anti-hipertensivos de primeira escolha em hipertensos sem complicações ou condições associadas.
\end{abstract}

Palavras-chave: hipertensão arterial, terapêutica, custobenefício

\section{Antihypertensive Treatment. Prescription and Cost of Drugs. Survey in a Tertiary Care Hospital}

Purpose - To study the most prescribed anti-hypertensive drugs, evaluating their agreement with established guidelines and drug cost.

Methods - One hundred and forty one (101 women, mean age $=53.3$ years) hypertensive patients who searched spontaneous attention were interviewed in a tertiary-care hospital. The inclusion criteria were previous diagnosis of hypertension and non cardiovascular complaints.

Results - The majority of the 107 (75.9\%) patients were on medical treatment. In those receiving monotherapy, thiazides were the most utilized drugs, followed by methyldopa, ACE inhibitors, calcium channel-blockers, and beta-blockers. The association with thiazides (26.3\%) followed the same preference. The second most prescribed drug, methyldopa, was the more expensive. Fifty percent of the patients purchased the drugs at their own expense.

Conclusion - A preference for prescription of expensive drugs for hypertension was detected in this sample in Brazil. This does not agree with major guidelines, mainly the $V$-JNC, which suggest thiazides and betablockers as first choice drugs for hypertensives with no complications or associated comorbidity.

Key-words: hypertension, therapy, cost-effectiveness

Arq Bras Cardiol, volume $71\left(n^{0} 1\right), 55-57,1998$

A hipertensão arterial (HA) é uma condição de alta prevalência em nosso meio, atingindo cerca de $20 \%$ da população adulta brasileira e apresentando freqüência maior nas camadas mais pobres da população ${ }^{1,2}$. Neste sentido,

Hospital das Clínicas da Faculdade de Medicina da USP

Correspondência: Paulo Andrade Lotufo - Disciplina de Clínica Geral - Av. Dr. Arnaldo, 455 S/1216 - 01246-903 - São Paulo, SP

Recebido para publicação em 5/1/98

Aceito em 1/4/98 tanto a eficácia como o custo da medicação devem ser considerados nas propostas de atendimento ao hipertenso.

A terapêutica medicamentosa mostrou-se eficaz no controle da pressão arterial (PA), contribuindo para a redução de eventos cardiovasculares ${ }^{3}$. Procedimentos como os "consensos" " realizados no Brasil ou guidelines como o The Joint National Committee on Prevention, Detection, Evaluation, and Treatment of High BloodPressure, na sua quinta versão (JNCV) ${ }^{5}$ são tentativas de uniformização e racionalização do atendimento ao hipertenso. 
Nosso objetivo é verificar, em amostra que procura hospital terciário com diagnóstico prévio de HA, qual o tipo de medicação em uso para o seu controle, modo e custo de sua aquisição.

\section{Métodos}

O inquérito foi realizado na triagem geral do Hospital das Clínicas da FMUSP, de outubro/95 a abril/96, onde foram entrevistados 141 pacientes consecutivos que procuraram atendimento médico em hospital público terciário por queixas variadas. Os diagnósticos de $\mathrm{HA}$ foram realizados em outros serviços, principalmente na rede pública de atenção médica da área metropolitana. Após atendimento de rotina na triagem e comprovação de ser hipertenso por dados de história, os pacientes foram encaminhados para entrevista onde respondiam a perguntas referentes ao uso de anti-hipertensivos. Foram excluídos todos os pacientes com queixas associadas ao aparelho cardiovascular, com encaminhamento para os serviços de cardiologia ou de nefrologia ou outros encaminhamentos que indicassem controle ineficaz da PA. Todos os pacientes selecionados atenderam à solicitação. Utilizaram-se sempre o mesmo horário do dia e os mesmos dias da semana para evitar viéses relativos a procura por determinada especialidade.

O questionário apresentava algumas questões prédeterminadas, como as relacionadas aos dados de identificação, origem e tempo de diagnóstico. A informação do medicamento anti-hipertensivo era formulada pela pergunta "qual o remédio que o senhor(a) está tomando nestes últimos dias para a pressão? Todos os remédios referidos foram listados e selecionados os associados ao tratamento anti-hipertensivo. A amostra apresentou nível sócio-econômico homogêneo, sendo representativa do Hospital, cuja clientela, estima-se, esteja no tercil inferior de renda.

Utilizou-se para cálculo do custo mensal de medicamentos, uma tabela de ampla aceitação no comércio varejista, o "Brasíndice”, editado em São Paulo.

\section{Resultados}

Conforme os dados apresentados na tabela I, foram entrevistados 141 pacientes hipertensos, sendo que $34(24,1 \%)$ não estavam em uso de medicamentos, apesar de tê-los adquirido. Os demais 107 (75,9\%) estavam em uso de monoterapia ou terapia combinada.

O grupo de medicamentos mais utilizados em esquema de monoterapia foi o dos diuréticos da classe dos tiazídicos, seguido pela metildopa, inibidores da enzima conversora da angiotensina (ECA), bloqueadores dos canais de cálcio e betabloqueadores, e os de esquemas de terapêutica combinada incluíam os diuréticos tiazídicos, sendo as associações mais freqüentes com metildopa, inibidores da ECA e bloqueadores dos canais de cálcio.

Cerca da metade $(n=79)$ dos pacientes recebeu medicamentos por meio de doação enquanto os demais $(n=62)$ adquiriram por compra direta no varejo, sem subsídio aparente.

\begin{tabular}{|c|c|c|c|}
\hline \multicolumn{4}{|c|}{$\begin{array}{l}\text { Tabela I - Características gerais dos pacientes com diagnóstico } \\
\text { médico prévio de hipertensão que procuraram atendimento em } \\
\text { hospital terciário. Aderência ao tratamento; tipo de medicação } \\
\text { utilizada e associação com tiazídicos e local de aquisição. }\end{array}$} \\
\hline Idade média (anos) & & 53,3 & \\
\hline Sexo masculino(\%) & & 26,2 & \\
\hline Aderência ao tratamento & \multicolumn{2}{|c|}{ número } & $\%$ \\
\hline Não & \multicolumn{2}{|c|}{34} & 24,1 \\
\hline Sim & \multicolumn{2}{|c|}{107} & 75,9 \\
\hline Terapêutica medicamentosa em uso & número & $\operatorname{parcial}(\%)$ & total $(\%)$ \\
\hline única & 65 & 100 & 60,7 \\
\hline tiazídicos & 35 & 53,9 & 32,8 \\
\hline metildopa & 10 & 15,4 & 9,4 \\
\hline inibidores da ECA & 9 & 13,8 & 8,4 \\
\hline bloqueadores canais de cálcio & 6 & 9,2 & 5,6 \\
\hline propranolol & 2 & 3,1 & 1,8 \\
\hline furosemida & 2 & 3,1 & 1,8 \\
\hline hidralazina & 1 & 1,5 & 0,9 \\
\hline dupla & 42 & 100 & 39,3 \\
\hline associada com tiazídico & 28 & 66,7 & 26,3 \\
\hline metildopa & 9 & 32,1 & 8,5 \\
\hline inibidores da ECA & 7 & 25,0 & 6,6 \\
\hline bloqueadores canais de cálcio & 7 & 25,0 & 6,6 \\
\hline propranolol & 3 & 10,8 & 2,8 \\
\hline prazosin & 2 & 7,1 & 1,8 \\
\hline outras associações & 13 & 33,3 & 13,0 \\
\hline Local de aquisição & \multicolumn{2}{|c|}{ número } & $\%$ \\
\hline farmácias & \multicolumn{2}{|c|}{62} & 43,9 \\
\hline postos de saúde & \multicolumn{2}{|c|}{27} & 19,2 \\
\hline farmácia hospitalar & \multicolumn{2}{|c|}{21} & 14,9 \\
\hline convênios & \multicolumn{2}{|c|}{3} & 2,2 \\
\hline outras locais & \multicolumn{2}{|c|}{7} & 4,9 \\
\hline não sabem informar & \multicolumn{2}{|c|}{21} & 14,9 \\
\hline
\end{tabular}

Com base na tabela de preços do primeiro semestre de 1996, calculou-se o custo de 30 dias de tratamento com dose mínima eficaz, segundo o preço dos principais consensos e utilizando-se da apresentação comercial de menor preço na ocasião. Constataram-se os seguinte custos mensais por grupo de medicamentos: diuréticos tiazídicos $(\mathrm{R} \$ 2,25)$, propranolol ( $\mathrm{R} \$ 2,37)$, nifedipina $(\mathrm{R} \$ 10,67)$, captopril $(27,5) \mathrm{e}$ metildopa $(\mathrm{R} \$ 44,55)$ (tab. I).

\section{Discussão}

O presente estudo apresenta as limitações inerentes a entrevistas realizadas em ambiente hospitalar, porque prescindiu da verificação exata do medicamento utilizado, obrigando ao paciente diferenciar o nome do medicamento hipertensivo em relação a outros medicamentos. A segunda limitação decorre do fato da clientela pesquisada estar procurando o hospital por outros motivos de saúde, o que poderia induzir viéses prévios na prescrição. A terceira limitação foi a que motivou a exclusão de pacientes que não sabiam ou não se lembravam do fármaco em uso. Um quarto óbice, que seria o alto grau de aderência ao tratamento observado (66\%), provavelmente foi devido à seleção rigorosa para entrevista. Por último, a distribuição de fármacos na rede pública, de forma errática, poderia estar influenciando a utilização de anti- 
hipertensivos. No entanto, inquéritos em hospitais que são referência podem representar instrumento útil de avaliação da assistência médica com baixo custo.

Uma pesquisa realizada em várias cidades brasileiras revelou que os medicamentos anti-hipertensivos mais utilizados em consultório de cardiologistas eram inibidores da ECA, seguidos de betabloqueadores, bloqueadores de canal de cálcio, diuréticos e metildopa, em ordem decrescente de preferência.

No presente trabalho, contudo, observou-se uma maior utilização da metildopa, isolada ou em associação com diuréticos. Tal fato merece destaque, uma vez que a metildopa foi o medicamento de maior custo entre os utilizados pelos pacientes da amostra.

A tendência de se utilizar inibidores da ECA e bloqueadores dos canais de cálcio, primeiro em consultórios e agora em clientela da rede pública, com baixo poder aquisitivo, coincide com o observado nos Estados Unidos, onde houve mudança no tratamento da HA nos últimos 10 anos. As supostas vantagens destes novos grupos de anti-hipertensivos se contrapõem aos seus custos mais altos e, conseqüentemente, menor aderência ao tratamento ${ }^{6,7}$.

Segundo as recomendações do JNC V todos esses medicamentos têm eficácia semelhante na redução da PA, po- rém ainda não se dispõem de estudos controlados a longo termo com o uso de inibidores da ECA, alfabloqueadores ou bloqueadores de canais de cálcio, surgindo então a preferência pelos tiazídicos e betabloqueadores. A prescrição dos outros grupos se justifica em condições especiais do hipertenso, como a presença de diabetes ou de insuficiência cardíaca.

Merecem destaque, no entanto, em nosso estudo: $1 \stackrel{\circ}{\circ}$ o fato que uma clientela com baixo poder aquisitivo, consuma medicamentos de maior custo, sendo que a metade dela os compra com recursos próprios; 2o, há nessa faixa da população uma utilização mais acentuada de metildopa. Por último, observa-se uma discrepância entre as medicações prescritas ao grupo estudado e aquelas preconizadas em consensos.

Novos estudos, com base populacional, avaliando a eficácia hipotensora e o impacto em desfechos clínicos, como o acidente vascular cerebral e o infarto do miocárdio, tornam-se necessários para uma melhor proposta de terapêutica anti-hipertensiva com perspectivas de ampliação do atendimento à maioria da população. A ação integrada dos órgãos governamentais, das sociedades científicas médicas e demais associações da sociedade civil devem se direcionar no sentido de proporcionar um esquema de atendimento ao hipertenso que seja efetivo e de baixo custo.

\section{Referências}

1. Duncan BB, Schmidt MI, Achutti AC et al - Socioeconomic distribution of noncommunicable disease risk factors in urban Brazil: the case of Porto Alegre. Bull PAHO 1993; 27: 337-49.

2. Lolio CA, Pereira JC, Lotufo PA et al - Hipertensão arterial e possiveis fatores de risco. Rev Saúde Publica. 1993; 27: 357-62.

3. Collins R, Peto R, MacMahon S et al - Blood pressure, stroke, and coronary heart disease. Part 2: short-term reductions in blood-pressure: overview of randomized drug trials in theri epidemiological context. Lancet 1990; 335: 827-35.

4. Segundo Consenso Brasileiro de Hipertensão Arterial. Arq Bras Cardiol 1994; 63: 333-47.
5. The Fifth Report of the Joint National Committee on Detection, Evaluation, and Treatment of High Blood Pressure (JNC V). Special article of the Joint National Committee on Detection, Evaluation and Treatment of High Blood Pressure. Arch Intern Med 1993; 153: 154-83.

6. Manolio TA, Cutler JA, Furberg CD, Psaty BM, Whelton PK, Applegate WB Trends in pharmacologic management of hypertension in the United States. Arch Intern Med 1995; 155: 829-37.

7. Siegel D, Lopez $\mathbf{J}$ - Trends in antihypertensive drug use in the United States. Do the JNC V recommendations affect prescribing? JAMA 1997; 278: $1745-8$. 